\title{
Fuzzy Logic Control of DFIG Small Hydropower Plant Connected to the Electrical Grid
}

\author{
Abdelkader Boudali $^{*}$, Karim Negadi ${ }^{1}$, Abderrahmane Berkani $^{1}$, Mohamed Boudiaf $^{2}$, Fabrizio Marignetti $^{3}$ \\ ${ }^{1}$ Laboratory of L2GEGI, Department of Electrical Engineering, Faculty of Applied Science, University of Tiaret, Tiaret, Algeria \\ ${ }^{2}$ Department of Electrical Engineering, University Ziane Achour of Djelfa, Djelfa, Algeria \\ ${ }^{3}$ Dipartimento di Ingegneria Elettrica e de ll'Informazione Università degli Studi di Cassino e del Lazio Meridionale, Cassino, \\ Italy
}

Corresponding Author Email: abdelkader.boudali@univ-tiaret.dz

https://doi.org/10.18280/ti-ijes.642-427

Received: 18 April 2020

Accepted: 29 May 2020

\section{Keywords:}

small hydropower plant, DFIG, vienna rectifier, multi-level inverter, fuzzy logic controller (FLC), power generation

\begin{abstract}
This paper presents the modeling, control, simulation and analysis of the dynamic small hydropower plant connected to the grid. Studied system comprises a small hydropower, a double fed induction generator (DFIG), and two converters linked by a DC bus. The whole is connected to the grid through a resistor-inductor filter. Hence, this paper proposed and discusses an efficient design based on a Fuzzy Logic Controller (FLC) applied side generator and side grid. This strategy provides good performance, such as insensitivity to non-linearity of system, the effects of disturbance regarding variation on the parameters, and the random nature of hydro turbine speed. Yet, the theory of classical sliding mode has faced the problem of chattering, which proved to be a major drawback. The considered control of hydropower plant system was implemented in the Matlab / Simulink, the results show the effectiveness of the proposed method. The prototype simulated can be realized in an experimental test bench based FPGA, DSP or Dspace controller board.
\end{abstract}

\section{INTRODUCTION}

Hydroelectric power plants (HPPs) are widely used and well-known energy production units all around the world. HPPs meet approximately $20 \%$ of the total electricity need of the world [1]. Generally, large power plants are preferred for electricity generation around the world, except for in a few countries. Therefore, it is not very common to produce electricity using small and microscale HPPs. This situation offers significant opportunities for small-scale and very-smallscale businesses $[1,2]$.

A hydropower generation system is a complex nonlinear power system including hydraulic, mechanical, electrical and magnetic subsystems. Nowadays, the size of HPPs and the structure complexity of systems have been increasing, especially in China. The proportion of electricity generated by intermittent renewable energy sources have also been growing. Therefore, the performance of HPPs in terms of frequency control is more and more important. The research on control strategies and dynamic processes of HPPs is of great importance. The frequency stability of hydropower units is a critical factor of power system security and power quality. The power response time for evaluating the frequency regulation quality, is also a key indicator.

In order to improve the quality of electric power produced by hydropower generators, a robust control insensitive to external perturbation is needed to control the production system. In the literature, a control strategy on the basis of proportional - integral (PI) controllers have been proposed to address the problem of maximum power extraction and to adjust the frequency and amplitude of voltage and current at the point of connection to the grid. The performances of PI controllers are limited. This limitation is due to nonlinearity of the system.

Therefore, there are a lot of intelligent nonlinear techniques used to overcome this difficulty, such as fuzzy logic and neural network that can be used for control of power converters

Fuzzy Logic Controller (FLC) does not require the knowledge of the exact model, it has the advantage to be robust and simple to design [3]. We implement this command strategy in order to meet the following specifications: good static precision in order to obtain optimal energy production and a unit power factor; good rejection of disturbances that can be frequent on such a system; robustness to any parametric variations of the system.

In this paper, a Mamdani fuzzy logic controller [4] is applied to control separately the rotor side converter (RSC) and the grid side converter (GSC).

Within this framework, the main interest of this control approach is to insert a control system for alleviating water hammer disturbances in order to control the power output of the hydro turbine and thus to limit it to its nominal value when the hydro water flow is too high, to ensure the extraction of maximum power even for low hydro turbine speed, to regulate both the reactive and active power independently, to maintain frequency and voltage at the connection point to the grid in qualifying beaches, and to ensure the operation of system at a unity power factor [5].

This proposed work is organized as follows: in section 2 , the modeling of the hydraulic turbine is defined. The system configuration and the models of components employed for each subsystem are described in section 3. In section 4 and 5, system is described with modeling focusing on the generator and associated controls.

Simulation results and subsequent discussions on performance evaluation are presented in section 6 . Section 7 
concludes the paper and also provides a glimpse of what should be done in future works.

\section{MODEL OF THE HYDRAULIC TURBINE}

The MHPP operation principle consists of transforming the hydraulic energy into electrical energy. Through a water tunnel, the water reaches a surge tank that supplies a penstock characterized by its height. At the output of this latter, the water rotates a hydraulic turbine which drives a synchronous generator. The generator produces electricity that feeds loads which can be composed from three or/and single-phase loads. Figure 1 shows the various components of the constituting hydropower plants.

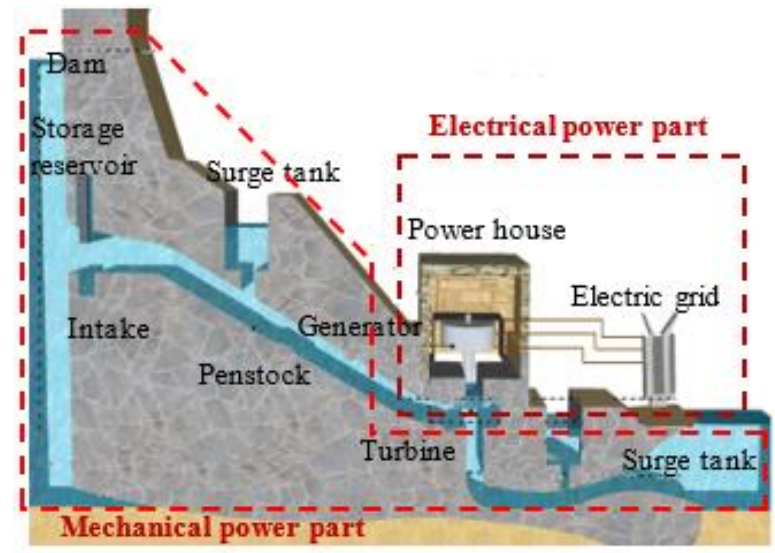

Figure 1. Hydroelectric power generation components

The Table 1 below shows the most widely hydropower categories. The table also shows the number of homes electrical energy needs met to put things in context.

Table 1. Hydropower plant category

\begin{tabular}{ccc}
\hline Hydro category & Power range & $\begin{array}{c}\text { Number of homes } \\
\text { powered }\end{array}$ \\
\hline Pico & $0 \mathrm{~kW}-5 \mathrm{~kW}$ & $0-5$ \\
\hline Micro & $5 \mathrm{~kW}-100 \mathrm{~kW}$ & $5-100$ \\
\hline Mini & $100 \mathrm{~kW}-1 \mathrm{MW}$ & $100-1,000$ \\
\hline Small & $1 \mathrm{MW}-10 \mathrm{MW}$ & $1,000-10,000$ \\
\hline Medium & $10 \mathrm{MW}-100 \mathrm{MW}$ & $10,000-100,000$ \\
\hline Large & $100 \mathrm{MW}+$ & $100,000+$ \\
\hline
\end{tabular}

The power absorbed from the hydraulic turbine depends on the net water head $H[m]$ and the water flow rate $Q_{\omega}\left[\mathrm{m}^{3} / \mathrm{s}\right][6]$ :

$$
p_{h}=\rho a H Q_{\omega}
$$

Hydraulic turbine efficiency $\eta$ is defined as the ratio of mechanical power transmitted by the shaft to the absorbed hydraulic power, which strongly affects the net output mechanical power $P_{m}[$ Watt $]$ :

$$
p_{m}=\eta \rho a H Q_{\omega}
$$

where, $\rho\left[\mathrm{kg} / \mathrm{m}^{3}\right]$ is the volume density of water, $a$ is the acceleration due to gravity $\left[\mathrm{m} / \mathrm{s}^{2}\right]$.

The mechanical torque could be given by:

$$
T_{m}=\frac{P_{m}}{\omega}
$$

where, $\omega[\mathrm{rad} / \mathrm{s}]$ represents the turbine rotation speed.

According to equations of turbine model, the plot of the hydraulic turbine characteristics is shown in Figure 2. This plot is in function according to several values of mechanical and speed turbine.

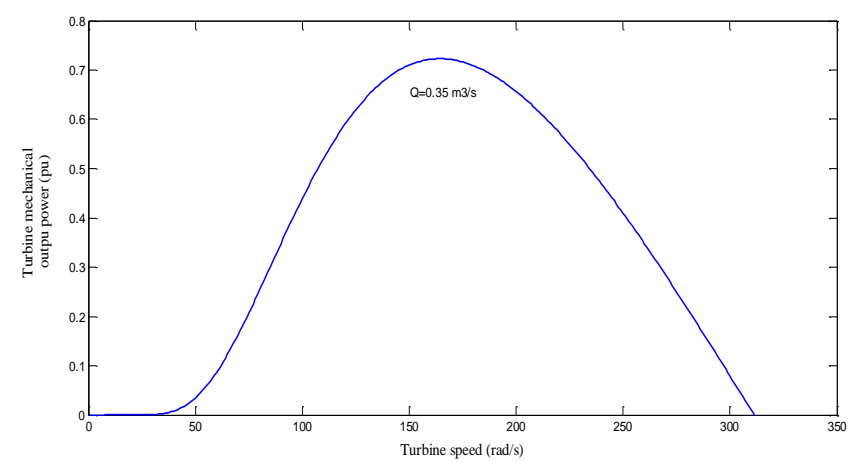

Figure 2. Hydralic turbine characteristic

\section{SYSTEM CONFIGURATION OF THE PROPOSED HYDRO PLANT SCHEMA}

The system configuration taking in consideration in this study is shown Figure 2 (a). As illustrated, the DFIG is an induction generator with the stator windings connected directly to the three-phase, constant-frequency grid and the rotor windings connected to back-to-back voltage source converters (VSCs), including a rotor-side converter (RSC) and grid-side converter (GSC) [6]. They are decoupled with a direct current (DC) link. Conventionally, the RSC controls the generator to regulate the active and reactive power, while the GSC controls the DC-link voltage to ensure DC voltage stability.

The power flow of the stator is always from hydraulic turbine to grid (Figure 3). However, the power flow of the rotor is dependent on the operating point:

- If the slip is negative (over-synchronous operation), it feeds power into the grid.

- If the slip is positive (sub-synchronous operation), it absorbs power from the grid.

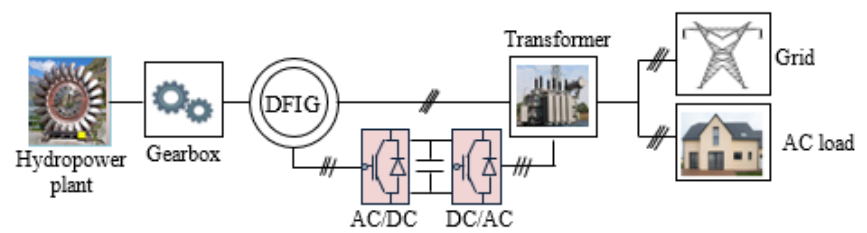

Figure 3. Configuration for variable speed of MHPP based DFGI

\section{ADVENCED MODELING}

\subsection{Double Fed Induction Generator model}

The electrical model of the DFIG in the d-q frame linked to the stator rotating field is given by the system [7]: 


$$
\left\{\begin{array}{l}
v_{s d}=R_{s} i_{s d}+\frac{d \psi_{s d}}{d t}-\omega_{s} i_{s q} \\
v_{s q}=R_{s} i_{s q}+\frac{d \psi_{s q}}{d t}+\omega_{s} i_{s d} \\
v_{r d}=R_{r} i_{r d}+\frac{d \psi_{r d}}{d t}-\omega_{r} i_{r q} \\
v_{r q}=R_{r} i_{s q}+\frac{d \psi_{r q}}{d t}+\omega_{r} i_{r d}
\end{array}\right.
$$

where:

$$
\left\{\begin{array}{l}
\psi_{s d}=L_{s} i_{s d}+M i_{r d} \\
\psi_{s q}=L_{s} i_{s q}+M i_{r q} \\
\psi_{r d}=L_{r} i_{r d}+M i_{s d} \\
\psi_{r q}=L_{r} i_{r q}+M i_{s q}
\end{array}\right.
$$

The electromagnetic torque is given by:

$$
T_{e m}=p M\left(i_{r d} i_{s q}-i_{r q} i_{s d}\right)
$$

By using the field oriented control approach we obtain:

$$
\left\{\begin{array}{l}
\frac{d i_{r d}}{d t}=\frac{1}{\sigma L_{r}}\left(v_{r d}-R_{r} i_{r d}+s \omega_{s} \sigma L_{r} i_{r q}-\frac{M}{L_{s}} \frac{d \psi_{s d}}{d t}\right) \\
\frac{d i_{r q}}{d t}=\frac{1}{\sigma L_{r}}\left(v_{r q}-R_{r} i_{r q}-s \omega_{s} \sigma L_{r} i_{r q}-s \omega_{s} \frac{M}{L_{s}} \psi_{s q}\right)
\end{array}\right.
$$

The expression of electromagnetic torque becomes:

$$
T_{e m}=-\frac{p M}{L_{s}} \psi_{s d} i_{r q}
$$

\subsection{Vienna rectifier}

The Vienna rectifier is an advantageous unidirectional PFC (power factor correction) rectifier with less number of active power switches, sinusoidal input current, and balanced output DC-link voltage, low voltage stress across switches, high switching operation and high efficiency [8]. The boost type rectifier is used for the wind, micro turbines, low voltage DC (LVDC), high voltage DC distribution (HVDC) and AC mains at the front side for higher voltages of $400 \mathrm{~V}-750 \mathrm{~V}-1500 \mathrm{~V}$.

It consists of 3-switches and 18-diodes with DC-link capacitor at the output. The current flows through the three MOSETs and the capacitors in the fully charged it. The phase current rises, through a MOSFETs, during that pulse period, charge the capacitor. When the MOSFETs is turned off, current through the diode upper or lower depending on direction of the current flow. By adjust the width of the pulse that turns ON the MOSFETs, corresponding line current is forced to be sinusoidal and in phase with the Voltage. When the MOSFETs is turned ON the corresponding phase is connected the line inductor, to the center point between the two output capacitors.

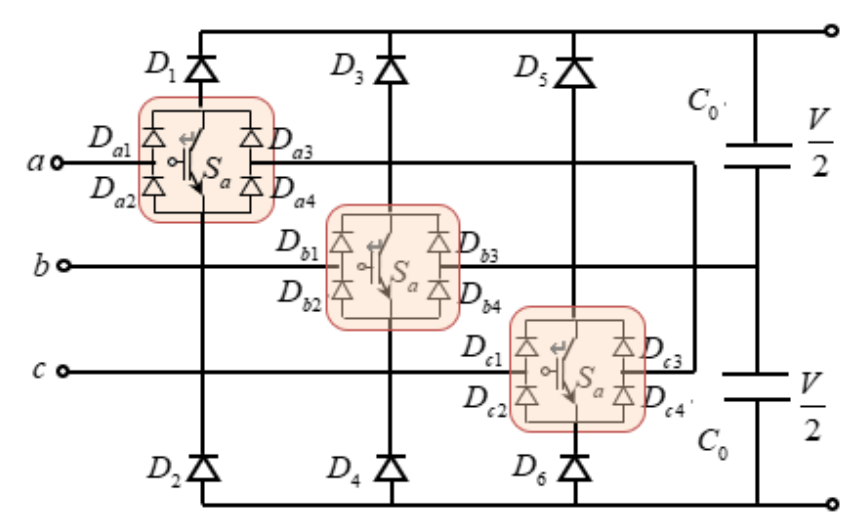

Figure 4. Topology of power Vienna rectifier

\subsection{Three-level inverter modeling}

The three level neutral point clamped inverter has many advantages over the conventional two level inverter, such as smoother waveform, less distortion, less switching frequency and low cost. The topology of a three level NPC inverter is shown in Figure 5.

The three level inverter has a total of 27 switching states $3^{3}$. When the upper switches $S_{a k l}, S_{a k 2}$ are in the on state, that corresponds to the state ' 1 '. When the lower switches $S_{a k 3}, S_{a k 4}$ are on, that corresponds to state ' -1 '. When the auxiliary switches $S_{a k 2}, S_{a k 3}$ are on, that results in state '0' [9].

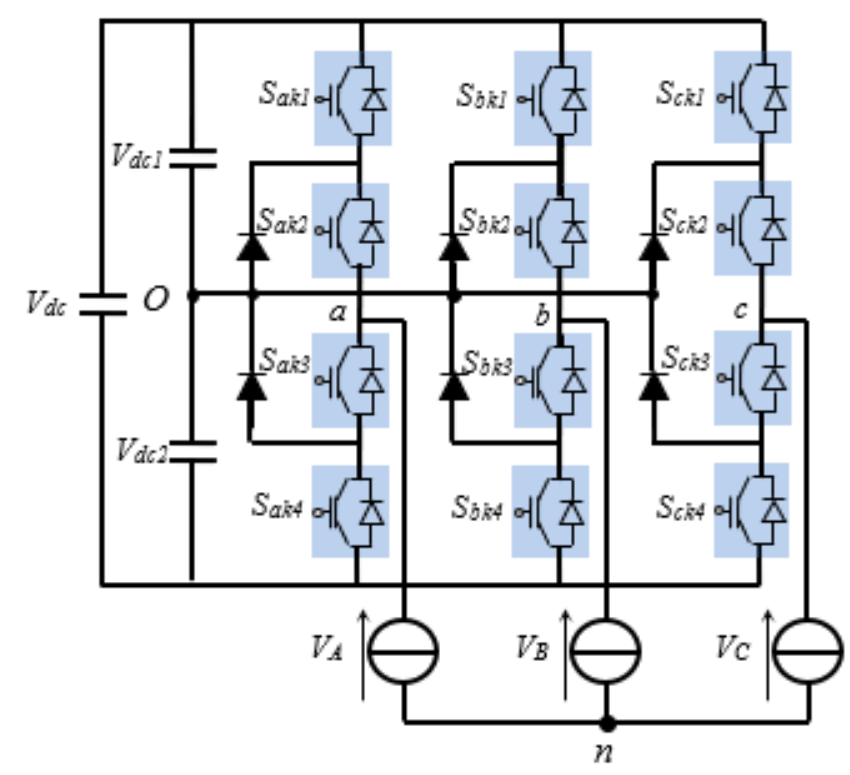

Figure 5. Power circuit of three-level inverter

The functions $\mathrm{F}_{\mathrm{km}}^{\mathrm{b}}$ of connection are given by:

$$
\left\{\begin{array}{l}
F_{k 1}^{b}=F_{k 1} \cdot \mathrm{F}_{k 2} \\
F_{k 0}^{b}=F_{k 3} \cdot \mathrm{F}_{k 4}
\end{array}\right.
$$

where, $m=1$ : the upper half arm and $m=0$ : the lower half arm.

The phase voltage $V_{A O}, V_{B O}, V_{C O}$ can be written as:

$$
\left\{\begin{array}{l}
V_{A O}=F_{11}^{b} V_{c 1}-F_{10}^{b} V_{c 2} \\
V_{B O}=F_{21}^{b} V_{c 1}-F_{20}^{b} V_{c 2} \\
V_{C O}=F_{31}^{b} V_{c 1}-F_{30}^{b} V_{c 2}
\end{array}\right.
$$


Simple output voltages are written as:

$$
\left[\begin{array}{l}
V_{A} \\
V_{B} \\
V_{C}
\end{array}\right]=\frac{1}{3}\left[\begin{array}{ccc}
2 & -1 & -1 \\
-1 & 2 & -1 \\
-1 & -1 & 2
\end{array}\right]\left\{\left[\begin{array}{c}
F_{11}^{b} \\
F_{21}^{b} \\
F_{31}^{b}
\end{array}\right] V_{c 1}-\left[\begin{array}{c}
F_{10}^{b} \\
F_{20}^{b} \\
F_{30}^{b}
\end{array}\right] V_{c 2}\right\}
$$

\subsection{Model of the grid}

The dynamic model of the grid connection in reference frame rotating synchronously with the grid voltage is given as follows:

$$
\left\{\begin{array}{l}
v_{d g}=v_{i d}-R_{g} i_{d g}-L_{d g} \frac{d i_{g d}}{d t}+L_{q g} \omega_{g} i_{q g} \\
v_{q g}=v_{i q}-R_{g} i_{q g}-L_{q g} \frac{d i_{q d}}{d t}-L_{d g} \omega_{g} i_{d g}
\end{array}\right.
$$

The DC-link system equation can be given by:

$$
C \frac{d v_{d c}}{d t}=\frac{3 v_{d g}}{2 v_{d c}} i_{d g}-i_{d c}
$$

where, $v_{d g}, v_{q g}$ are the direct and quadrature components of the grid voltages, $v_{i d}, v_{i q}(\mathrm{~V})$ are the inverter voltages components, $R_{g}, L_{d g}, L_{q g}$ are the resistance, the direct and quadrature grid inductance, respectively, $i_{d g}, i_{q g}(\mathrm{~A})$ are the direct and quadrature components of the grid currents, respectively, $v_{d c}$ is the DC-link voltage, $i_{d c}$ is the grid side transmission line current and $C$ is the DC-link capacitor.

The power equations in the synchronous reference frame are given by [10]:

$$
\left\{\begin{array}{l}
P_{g}=\frac{3}{2}\left(v_{d g} i_{d g}+v_{q g} i_{q g}\right) \\
Q_{g}=\frac{3}{2}\left(v_{d g} i_{d g}-v_{q g} i_{q g}\right)
\end{array}\right.
$$

After orienting the reference frame along the grid voltage, $v_{q g}$ equals to zero by aligning the d-axis. Then, the active and reactive power can be obtained in this new reference from the following equations [10]:

$$
\left\{\begin{array}{l}
P_{g}=\frac{3}{2} v_{d g} i_{d g} \\
Q_{g}=\frac{3}{2} v_{d g} i_{q g}
\end{array}\right.
$$

\section{FUZZY POWER CONTROL OF THE DFIG}

Fuzzy logic is an extension of classical logic that allows approaching the human reasoning that makes use of the tolerance, uncertainty, imprecision and flexibility in the decision-making process [11]. It was introduced by L. Zadeh in the mids of $60 \mathrm{~s}$. In recent years, the number and variety of applications of fuzzy logic have increased significantly.

\subsection{Control of the rotor side converter (RSC)}

When the DFIG is connected to an existing grid, this connection must be established in the following three steps:

- The first step is the synchronisation of the stator voltages with the grid voltages, which are used as a reference.

- The second step is the stator connection to this grid. After that, the connection can be effectively established. Once this connection is achieved.

- The third step is the regulation of the transit of the power between the DFIG and the grid a d-q referenceframe synchronized with the stator flux is employed [12]. By setting the quadratic component of the stator to the null value as follows:

$$
\left\{\begin{array}{l}
\psi_{s d}=\psi_{s} \\
\psi_{s q}=0
\end{array}\right.
$$

The stator voltage in Eq. (4) can be simplified as:

$$
\left\{\begin{array}{l}
v_{s d}=0 \\
v_{s q}=v_{s}=\omega_{s} \psi_{s d}
\end{array}\right.
$$

Then, with regard to (16), the fluxes are simplified as indicated below:

$$
\left\{\begin{array}{l}
\psi_{s d}=L_{s} i_{s d}+M i_{r d} \\
\psi_{s q}=L_{s} i_{s q}+M i_{r q}=0
\end{array}\right.
$$

The adaptation of the relations (14) to the chosen system of axes and the simplifying hypothesis considered in our case gives [13]:

$$
\left\{\begin{array}{l}
P_{s}=-v_{s} \frac{M}{L_{s}} i_{s q} \\
Q_{s}=\frac{v_{s} \psi_{s}}{L_{s}}-\frac{v_{s} M}{L_{s}} i_{s d}
\end{array}\right.
$$

Therefore, power control is achieved by regulating the $\mathrm{d}$ and $\mathrm{q}$ rotor currents:

$$
\left\{\begin{array}{l}
v_{r d}=R_{r} i_{r d}-L_{r} \omega_{r} \sigma i_{r q}+L_{r} \sigma \frac{d i_{r d}}{d t} \\
v_{r q}=R_{r} i_{r q}+L_{r} \omega_{r} \sigma i_{r d}+\frac{M \omega_{r}}{L_{s}} \psi_{s d}+L_{r} \sigma \frac{d i_{r q}}{d t}
\end{array}\right.
$$

The plant for the current control loops is given in (18) and based on the controller output voltages $v_{r d}^{*}$ and $v_{r q}^{*}$ :

$$
F(s)=\frac{i_{r d}(s)}{v_{r d}^{*}(s)}=\frac{i_{r q}(s)}{v_{r q}^{*}(s)}=\frac{v_{s} M}{R_{r} L_{s}+s L_{r} L_{s} \sigma}
$$

The controller compensation voltages are given by:

$$
\left\{\begin{array}{l}
e_{d}=g \omega_{s} \frac{M}{L_{s}} \psi_{s} \\
e_{q}=\frac{v_{s} R_{r}}{\omega_{s} M}
\end{array}\right.
$$


The block diagram of the simplified system to be controlled is presented in Figure 6.

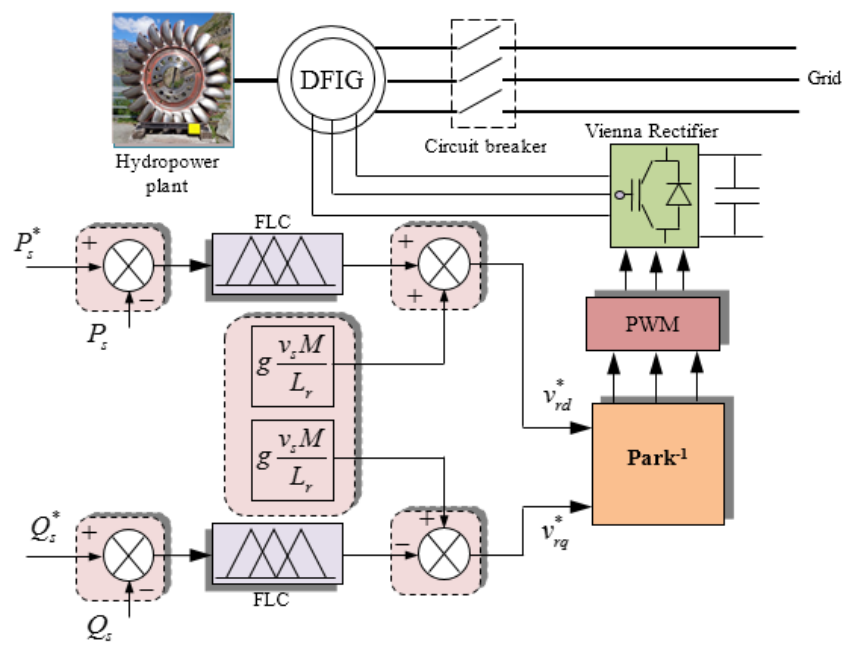

Figure 6. Schematic of the fuzzy logic control of the rotor side converter

\subsection{Control of the grid side converter (GSC)}

The GSC voltages in its d-q components are obtained by the typical configuration of control strategy are given by [14]:

$$
\left\{\begin{array}{l}
\frac{d i_{d g}}{d t}=-\frac{v_{d g}}{L_{g}}-\frac{R_{g}}{L_{g}} i_{d g}+\omega_{s} i_{q g}+\frac{v_{s d}}{L_{g}} \\
\frac{d i_{q g}}{d t}=-\frac{v_{q g}}{L_{g}}-\frac{R_{g}}{L_{g}} i_{q g}-\omega_{s} i_{d g}+\frac{v_{s q}}{L_{g}}
\end{array}\right.
$$

Applying the Laplace transforms to the above two equations:

$$
\left\{\begin{array}{l}
v_{d g}=\left(R_{g}+s L_{g}\right) i_{d g}-\omega_{s} L_{g} i_{q g}+v_{d g} \\
v_{q g}=\left(R_{g}+s L_{g}\right) i_{q g}+\omega_{s} L_{g} i_{d g}+v_{q g}
\end{array}\right.
$$

and:

$$
\left\{\begin{array}{l}
v_{d}=\left(R_{g}+s L_{g}\right) i_{d g} \\
v_{q}=\left(R_{g}+s L_{g}\right) i_{q g}
\end{array}\right.
$$

The plant for the current control loops is given by:

$$
F(s)=\frac{i_{q g}}{v_{q}}=\frac{i_{d g}}{v_{d}}=\frac{1}{\left(R_{g}+s L_{g}\right)}
$$

$i_{d g}$ and $i_{q g}$ demand being derived respectively from the DC link voltage error through a FLC and the displacement factor on the supply side of the inductor.

Substituting (26) in (25), the reference direct and quadrature control signals can be given as:

$$
\left\{\begin{array}{l}
v_{d}^{*}=-v_{d}+\omega_{s} L_{g} i_{q g}+v_{s d} \\
v_{q}^{*}=-v_{q}-\omega_{s} L_{g} i_{d g}+v_{s q}
\end{array}\right.
$$

To control the DC bus voltage $\mathrm{VC}$, a regulator must be implemented to maintain this voltage constant regardless of the current flow rate on the capacitor. The DC bus equation can be written as follows [15]:

$$
P_{c}=c v_{c} \frac{d v_{c}}{d t}=P_{g}-P_{r}
$$

where, $P_{r}$ and $P_{g}$ are the active powers at RSC and GSC side, respectively.

To control the dc voltage, we control the $P_{c}$ in the capacitor by adjusting the power $P_{g}$.

The FLC structure for the GSC and the DC-link voltage control is shown in Figure 7.

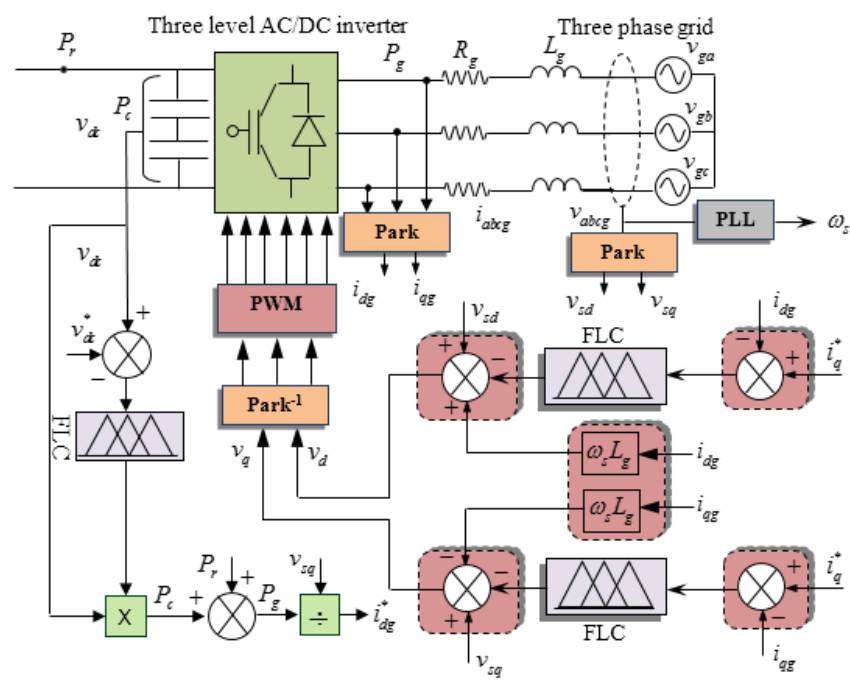

Figure 7. Schematic of grid side converter based fuzzy logic control

\subsection{Fuzzy Logic Controller (FLC) based converter design}

The rule base adjusts the reference voltage of the NPC inverter based upon the changes in the input of the FLC. The number of rules can be set as desired. The rule base includes 49 rules, which are based upon the seven membership functions of the input variables.

Table 2 shows the rule base for the FLC.

Table 2. Rule base of fuzzy logic control

\begin{tabular}{|c|c|c|c|c|c|c|c|}
\hline \multirow{2}{*}{$\boldsymbol{e}$} & \multicolumn{9}{|c|}{$\boldsymbol{d} \boldsymbol{e}$} \\
\cline { 2 - 8 } & LN & MN & SN & Z & SP & MP & LP \\
\hline LN & LP & LP & LP & MP & MP & SP & Z \\
\hline MN & LP & MP & MP & MP & SP & Z & SN \\
\hline SN & LP & MP & SP & SP & Z & SN & MN \\
\hline Z & MP & MP & SP & Z & SN & MN & MN \\
\hline SP & MP & SP & Z & SN & SN & MN & LN \\
\hline MP & SP & Z & SN & MN & MN & MN & LN \\
\hline LP & Z & SN & MN & MN & LN & LN & LN \\
\hline
\end{tabular}

where,

LP: Large Positive,

MP: Medium Positive,

SP: Small Positive,

Z: Zero,

SN: Small Negative,

MN: Medium Negative, 
LN: Large Negative.

The basic fuzzy sets of membership functions for the variables are as shown in Figure 8. The fuzzy variables are expressed by linguistic variables 'large negative (LN)', 'medium negative (MN)', 'small negative (SN)', 'zero (Z)', 'small positive (SP)', 'medium positive (MP)', 'large positive (LP)', for all three variables.

The knowledge base consists of a data base with the necessary linguistic definitions and control rule set. The rule set of knowledge base consists of some fuzzy rules that define the relations between inputs and outputs.

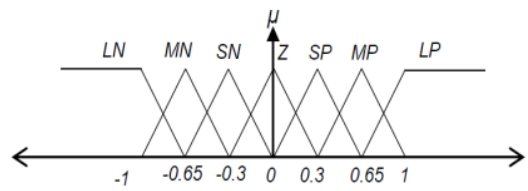

Figure 8. Membership function for input variables

\section{SIMULATION RESULTS AND DISCUSSIONS}

Simulations validation of the global control scheme presented throughout section 5 is carried out on the $2 \mathrm{MW}$ DFIG where the electric parameters under consideration are collected in Table 2. The frequency of the AC grid was $50 \mathrm{~Hz}$ (characteristics of the DC-AC converter and the grid illustrated in Table 3).

To evaluate and test the proposed control technique for a complete model of hydropower plant based DFIG with power loop of current, active and reactive power and dc voltage using fuzzy logic controllers, a simulation was performed under the MATLAB / Simulink.

The amount of power generated/supplied by the hydroelectric turbine depends on the variable water flow rated $\mathrm{Q}=0.8 \mathrm{~m}^{3} / \mathrm{s}$ at the time between $\mathrm{t}=[1-5 \mathrm{~s}], \mathrm{Q}=0.6 \mathrm{~m}^{3} / \mathrm{s}$ at the time between $\mathrm{t}=[15.5-20 \mathrm{~s}]$ and $\mathrm{Q}=0.9 \mathrm{~m}^{3} / \mathrm{s}$ at the time between $\mathrm{t}=[20.5-25 \mathrm{~s}]$ respectively (Figure $9(\mathrm{a})$ ).

As the water flow changes, Figure 9 (b) show that the mechanical torque ripple is smaller. The synchronous speed of the rotor display in Figure 9 (c) was 1 pu. The mechanical speed of the generator follows the reference that is determined by control strategy to capture the maximum hydroelectric power. The mechanical speed has a faster response.
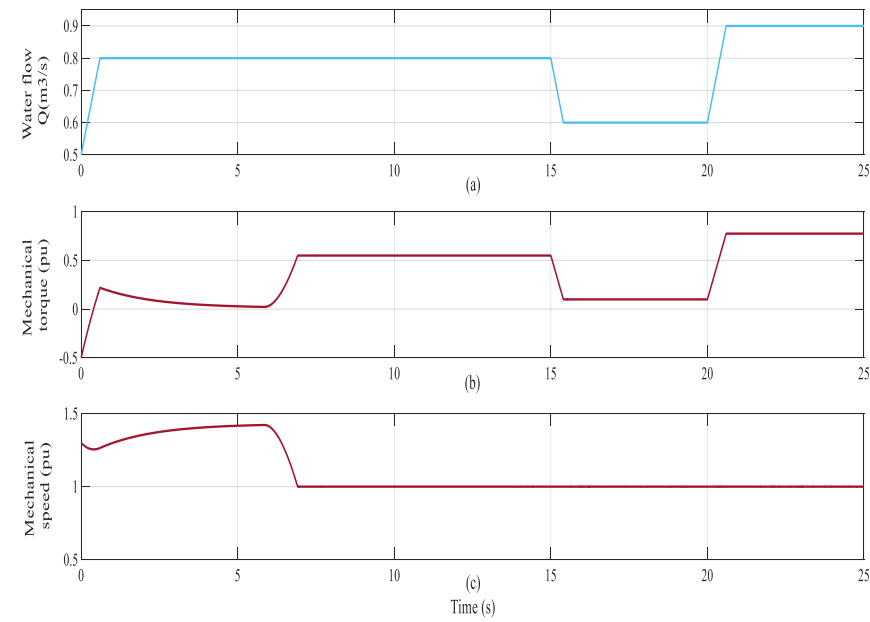

Figure 9. Hydropower plant mechanical characteristics output under varying water flow
Figure 10 displayed in $\mathrm{pu}$ the electromagnetic torque developped by the generator. This waveform of torque present less chattering and respond appropriately to the proposed variation.

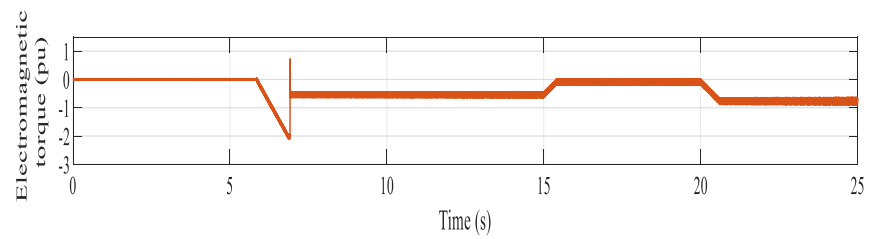

Figure 10. Electromagnetic torque of the DFIG
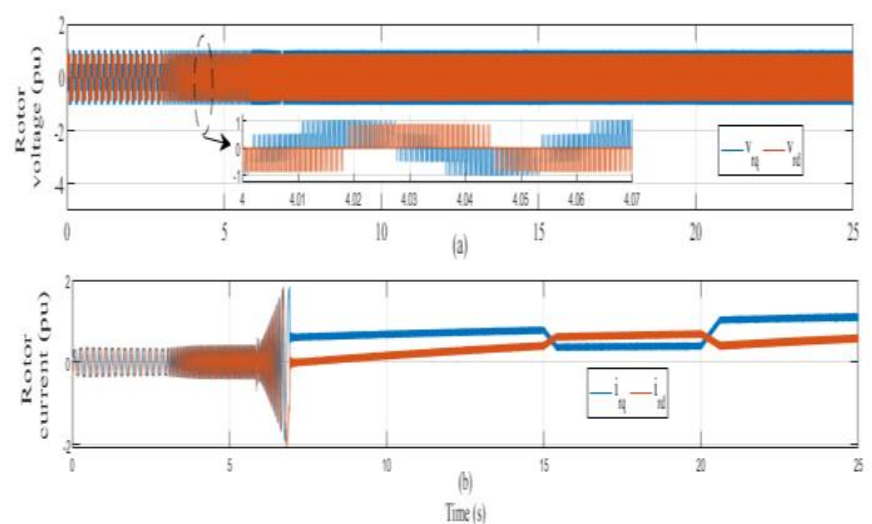

Figure 11. Dynamic response of the DFIG of the proposed control strategy: (a) and (b) rotor voltage and current $d, q$ components respectively

Figure 11 (a) and (b) displayed respectively the d, q components of the rotor voltage and current and its zoom. It seen that these curves are the same paces with the trend of the hydraulic caracteristics and are affected by the instants of variations.

Figure 12 shows that the rotor flux component ripple using fuzzy logic controller method is smaller. Both parts of the figure show average amplitude of the rotor flux around $1 \mathrm{pu}$. The maximum deviation of the rotor flux in Figure 12 is 0.15 pu. That means the DFIG system using the FLC method has reduced the deviation of the rotor flux by $44.4 \%$.

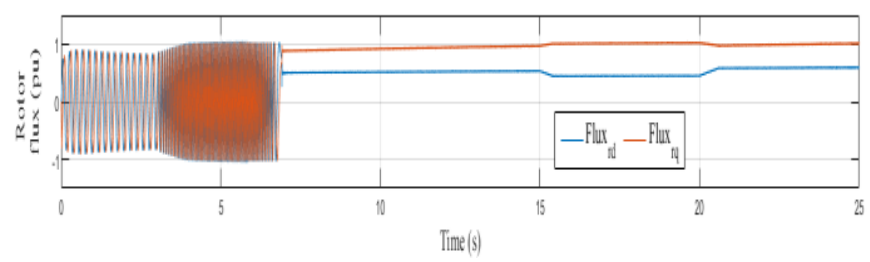

Figure 12. Dynamic response of the DFIG of the proposed control strategy: rotor flux $d, q$ components

The simulation results of the second part in the Figure 13 at 19 displays the (DC link voltage, grid and generator frequency, grid current and voltage, active and reactive power, and the THD and spectrum analysis) on the grid side converter, controlled via the proposed FLC.

One important performance measure of a DFIG system is the voltage of DC-link between the rotor-side converter and the grid-side converter, as shown is Figure 13. The DC bus voltage, the generator and the grid frequency responses with 
FLC controller remain outstandingly insensible to the variation of the hydraulic water flow with smaller ripple.

This is due its high ability to reject the disturbances and uncertainties, FLC algorithm do not depend on the system parameters, such as that the highest robustness can be achieved. Figure 14 (a) and (b) display respectively the generator and grid frequency, the two frequencies are synchronized at $\mathrm{t}=7$ sec.

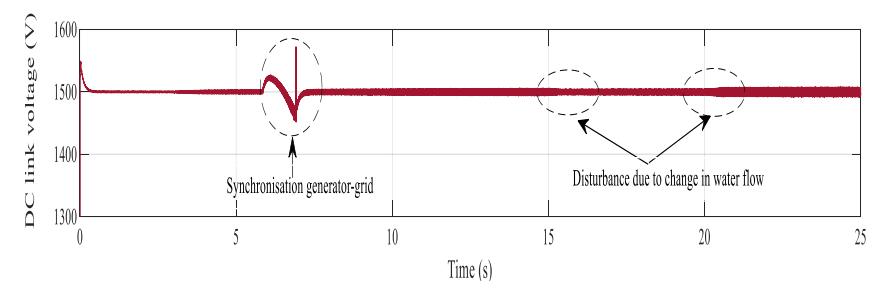

Figure 13. Dynamic response of proposed control: dc link voltage
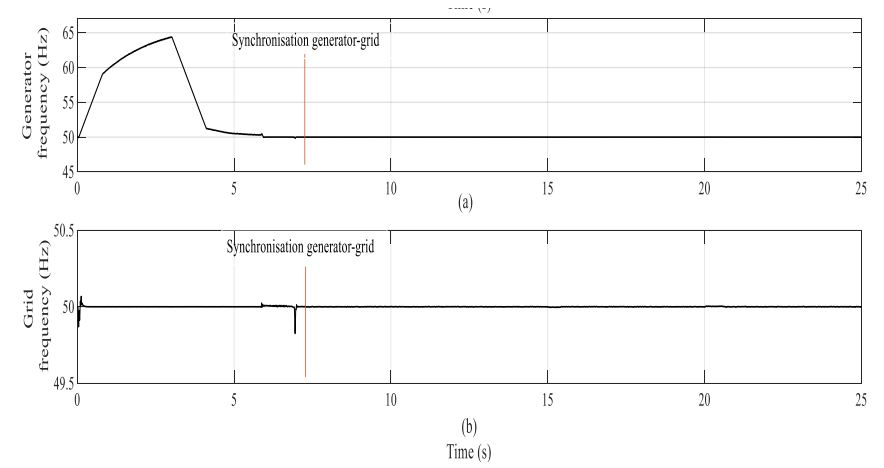

Figure 14. Dynamic response of proposed control: (a) generator frequency, (b) grid frequency

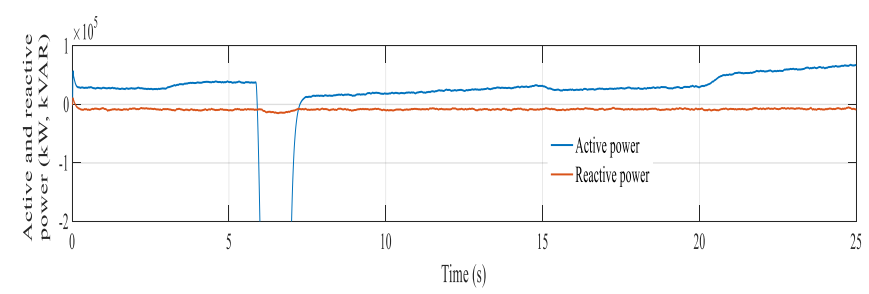

Figure 15. Dynamic response of the proposed control strategy: stator active and reactive powers

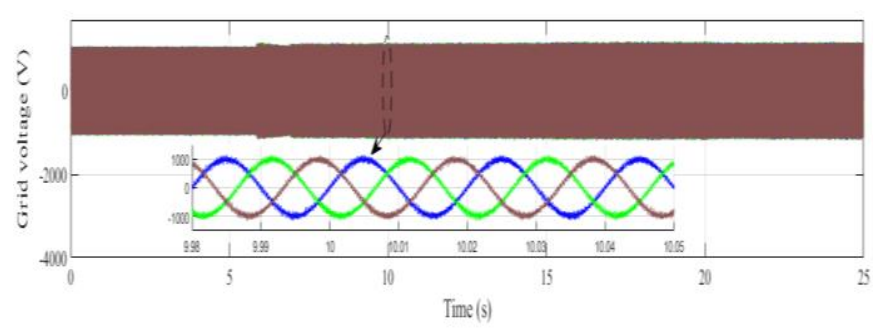

Figure 16. Dynamic response of the proposed control strategy: grid voltage

Figure 15 show the variations of active and reactive power, the active power keeps its nominal value of $1.5 \mathrm{MW}$ and the reactive power remains near zero MVAR with slight disturbances appear at the instants of variations in water flow and with fast response time.
The Figure 16 and Figure 17 respectively show the current and the voltage of the load with their zoom, they are purely sinusoidal which shows the efficiency of the control strategy applied to the converter which gives a better quality of energy to the grid.

In Figure 18, hence the change in power flow direction can be seen as $180^{\circ}$ phase shift between the grid voltage and grid current

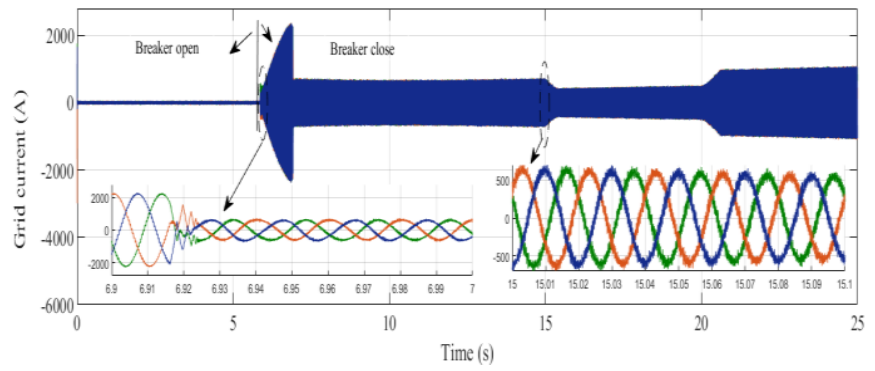

Figure 17. Dynamic response of the proposed control strategy: grid current

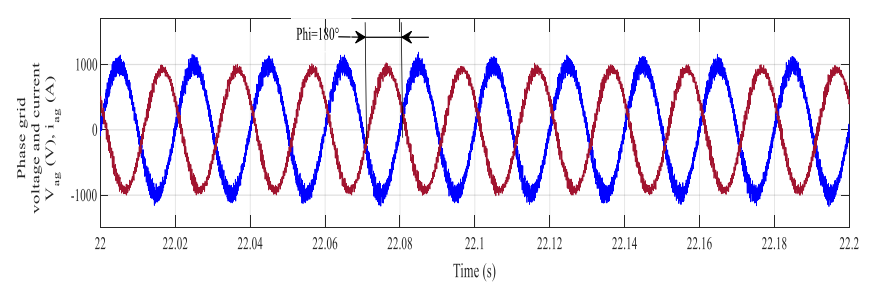

Figure 18. Phase grid voltage and current
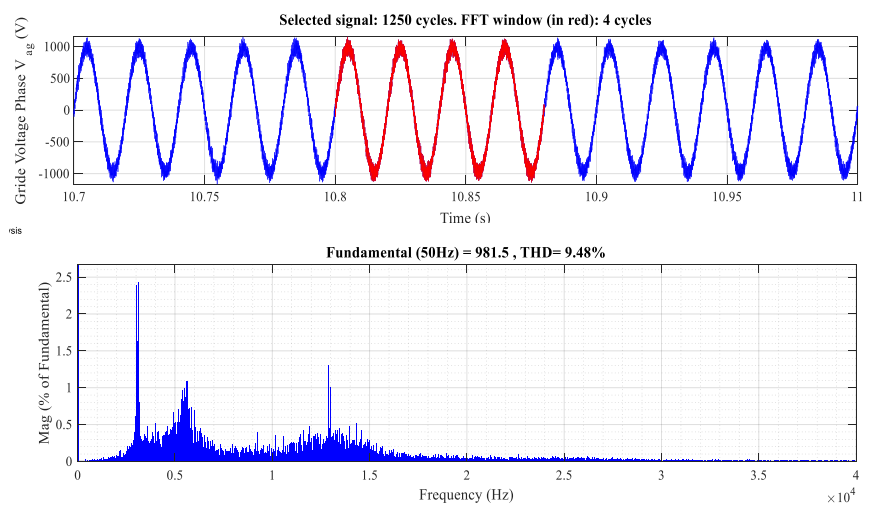

(a)

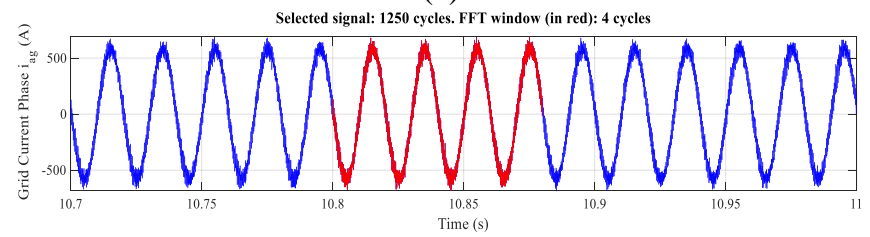

Fundamental $(50 \mathrm{~Hz})=581, \mathrm{THD}=12.86 \%$

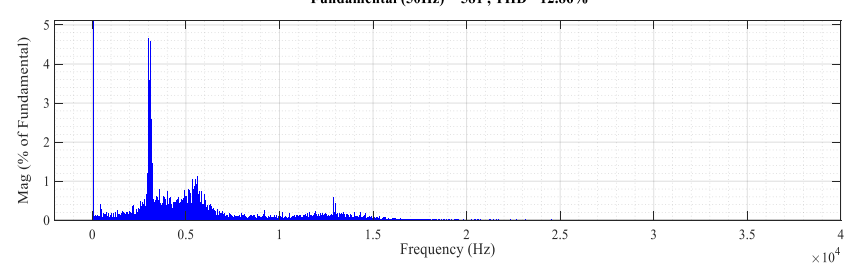

(b)

Figure 19. THD and spectrum analysis: (a) grid voltage, (b) grid current 
Figure 19 (a) and (b) illustrates respectively a sample waveform of the grid current and voltage for phase A. To see the efficiency of the proposed control strategy FLC, a harmonic frequency spectrum estimated by means of an FFT analysis and total harmonic distortion (THD) of the grid current and voltage are show in Figure 19 where each harmonic amplitude is expressed in percentage of the amplitude of the fundamental. THD is about $9.73 \%$ and $9.79 \%$ respectively.

These harmonic rates obtained are very satisfactory for this studied voltage level of $1200 \mathrm{~V}$.

Extensive simulations under different working conditions are performed for the considered hydroelectric dynamic process. The achieved results indicate that the proposed methodology is effective to accurately describe the hydroelectric power plant nonlinear dynamics as well as to design a hydraulic turbine speed control system.

\section{CONCLUSIONS}

In this paper, a doubly fed induction generator as the power conversion system in hydropower turbines is analysed by vector controlled for better control of the grid while injecting the required active power of the system.

A design of an optimal fuzzy logic controller for turbine on 1.5 MW DFIG is used to improve the performances of the whole system under external disturbance.

The proposed approach used in the conversion chain compared with conventional modulation technique gives a minimum THD value and powers ripples and it considerably reduces chatter active power and electromagnetic torque, avoiding the damage of excessive mechanical stress hydroelectric turbine.

Compared to the conventional standard DFIG control technique, the proposed method is more stable and reliable, has better dynamic performance, and demonstrates superior behavior particularly under the ac system bus voltage control mode.

The proposed methods can be implemented easily with FPGA, DSP or Dspace controller board platform.

\section{REFERENCES}

[1] Baoling, G., Bacha, S., Alamir, M., Amgad, M. (2018). Variable speed micro-hydro power generation system: Review and experimental results. Symposium de Génie Electrique (SGE 2018), Nancy, France.

[2] Yang, W.J. (2017). Dynamic processes and active power control of hydropower plants. Thesis, Uppsala University.

[3] Chenna, A., Aouzellag, D., Ghedamsi, K. (2020). Study and control of a pumped storage hydropower system dedicated to renewable energy resources. Journal Européen des Systèmes Automatisés, 53(1): 95-102. https://doi.org/10.18280/jesa.530112

[4] Rached, B., Elharoussi, M., Abdelmounim, E. (2019). Fuzzy logic control for wind energy conversion system based on DFIG. International Conference on Wireless Technologies, Embedded and Intelligent Systems (WITS), Fez, Morocco, pp. 1-6. https://doi.org/10.1109/WITS.2019.8723722

[5] Guan, Y., Quintero, J.C.V., Guerrero, J.M., Wang, Y., Feng, W. (2015). Frequency stability of hierarchically controlled hybrid photovoltaic-battery-hydropower microgrids. IEEE Transactions on Industry Applications, 51(6): 4729-4742. https://doi.org/10.1109/TIA.2015.2458954

[6] Zhou, W. (2017). Modeling, control and optimization of a hydropower plant. university college of southeast norway faculty of technology, natural sciences and maritime sciences. PhD dissertation in Process, Energy and Automation Engineering, Doctoral dissertation no. 33.

[7] Beltran, B., Benbouzid, M.E., Ahmed-Ali, T. (2012). Second-order sliding mode control of a doubly fed induction generator driven wind turbine. IEEE Transactions on Energy Conversion, 27(2): 261-269. https://doi.org/10.1109/TEC.2011.2181515

[8] Hao, C., David, N., Dionysios, C., Aliprantis, C. (2012). Analysis of permanent-magnet synchronous generator with vienna rectifier for wind energy conversion system. IEEE Transactions on Sustainable Energy, 4(1): 154-163. https://doi.org/10.1109/TSTE.2012.2208660

[9] Berkani, A., Negadi, K., Allaoui, T., Marignetti, F. (2019). Sliding mode control of wind energy conversion system using dual star synchronous machine and three level converter. Tecnica Italiana-Italian Journal of Engineering Science, 63(2-4): 243-250. https://doi.org/10.18280/ti-ijes.632-418

[10] Krim, Y., Abbes, D., Krim, S. , Mimouni, M.F. (2018). Classical vector, first-order sliding mode and high-order sliding-mode control for a grid-connected variable speed wind energy conversion system: A comparative study. Wind Engineering, 42(1): 16-37. https://doi.org/10.1177/0309524X17723202

[11] Benbouhenni, H., Boudjema, Z., Belaidi, A. (2019). DPC based on ANFIS super-twisting sliding mode algorithm of a doubly-fed induction generator for wind energy system. Journal Européen des Systèmes Automatisés, 53(1): 69-80. https://doi.org/10.18280/jesa.530109

[12] Li, S., Haskew, T.A., Williams, K.A., Swatloski, R.P. (2012). Control of DFIG wind turbine with direct-current vector control configuration. IEEE Transactions on Sustainable $\quad$ Energy, $3(1)$ : 1-11. https://doi.org/10.1109/TSTE.2011.2167001

[13] Moussa, O., Benguesmia, H., Abdessemed, R., Benaggoune, S. (2019). High-order sliding mode control of a grid-connected brushless doubly fed induction generator. International Conference on Mechanics and Energy, Monastir, Tunisia.

[14] Boubzizi, S., Abid, H., El hajjaji, A., Chaabane, M. (2018). Comparative study of three types of controllers for DFIG in wind energy conversion system. Springer, Protection and Control of Modern Power Systems, 3: 21. https://doi.org/10.1186/s41601-018-0096-y

[15] Anjana, J., Shankar, S., Vanitha, V. (2018). Power generation using permanent magnet synchronous generator (PMSG) based variable speed wind energy conversion system (WECS): An overview. Journal of Green Engineering, 7(4): 477-504. https://doi.org/10.13052/jge1904-4720.742

\section{NOMENCLATURE}

$v_{d g}, v_{q g}$
Grid peak voltages in dq-frame [V] are the $\mathrm{d}, \mathrm{q}$ components of the stator voltage 
are the $\mathrm{d}, \mathrm{q}$ components of the rotor voltage

Voltage inverter d, q components [V]

Grid peak currents in dq-frame[A]

are the $\mathrm{d}, \mathrm{q}$ components of the stator current are the $\mathrm{d}, \mathrm{q}$ components of the rotor current are the $\mathrm{d}, \mathrm{q}$ components of the stator flux are the $\mathrm{d}, \mathrm{q}$ components of the rotor flux are the stator and rotor resistance are the stator and rotor inductance is the magnetizing inductance

\section{Slip of DFIG}

are the synchronous and rotor angular speed is the slip angular speed

Input filter resistance $[\Omega]$

Input filter inductance $\mathrm{d}$, q components [mH]

Grid angular frequency [rad/s]

Dc bus voltage [V]

Reference Dc bus voltage [V]

Dc bus current [A]

DC link capacitor $[\mathrm{mF}]$

Load resistance $[\Omega]$

Grid active power [kW]

Grid active power reference $[\mathrm{kW}]$

The power in capacitor

Grid active power

The active power at rotor side converter

The mechanical power

Number of pole pairs in a generator

Grid reactive power [kVAR]

Grid reactive power reference [kVAR]

Water flow $\left[\mathrm{m}^{3} / \mathrm{s}\right]$

Switching state

Electromagnetic torque [Nm]

The mechanical torque $[\mathrm{Nm}]$

Sampling time $[\mu \mathrm{s}]$

Grid frequency $[\mathrm{Hz}]$

Present sampling instant in discrete-time frame

Future sampling instant in discrete-time frame

Past sampling instant in discrete-time frame

Water head [m]

Grid power factor angle (angle between grid

voltage and current) [degree]

Hydraulic turbine efficiency

The volume density of water $\left[\mathrm{kg} / \mathrm{m}^{3}\right]$
$\mathrm{AC}$

DC

DFIG

DSP

FLC

FPGA

GSC

HPP

HVDC

LVDC

MOSFET

NPC

PI

PPC

pu

PWM

RCS

THD

VSC

\section{APPENDIX}

Table 2. DFIG data used for simulation

\begin{tabular}{cc}
\hline Components & Rating values \\
\hline Rated power & $1600 \mathrm{kVA}$ \\
Rated voltage Vrms & $1200 \mathrm{~V}$ \\
Stator and rotor resistance & $0.01379,0.007728 \mathrm{pu}$ \\
Stator, rotor and mutual inductance & $0.0955,0.0955,2.416 \mathrm{pu}$ \\
Inertia constant & $1.1 \mathrm{pu}$ \\
Friction factor & $0.008726 \mathrm{pu}$ \\
Pole pairs & 2 \\
\hline
\end{tabular}

Table 3. DC-AC inverter and grid parameters

\begin{tabular}{cc}
\hline Components & Rating values \\
\hline Rated current & $1000 \mathrm{~A}$ \\
Frequency & $50 \mathrm{~Hz}$ \\
Rated voltage Vrms & $1200 \mathrm{~V}$ \\
Capacity rating & $0.01 \mathrm{~F}$ \\
\hline
\end{tabular}

